\title{
Treatment persistence of biologics among patients with psoriatic arthritis
}

\author{
Amir Haddad ${ }^{1 *}$, Tal Gazitt ${ }^{1}$, Ilan Feldhamer ${ }^{2}$, Joy Feld ${ }^{1}$, Arnon Dov Cohen²,3 Idit Lavi ${ }^{4}$, Faten Tatour ${ }^{5}$,
} Irena Bergman ${ }^{5}$ and Devy Zisman ${ }^{1,6^{*}}$

\begin{abstract}
Background: Persistence of biologic therapy in psoriatic arthritis (PsA) patients is an important factor in individualized patient treatment planning and healthcare policy and guideline development.
\end{abstract}

Objective: To estimate the persistence of biologic agents prescribed to PsA patients in a real-life setting as well as factors associated with improved biologic drug survival in these patients.

Methods: Patients with PsA from a large healthcare provider database with at least two consecutive dispensed prescriptions of a biologic agent indicated for PsA from January 1, 2002, until December 31, 2018, were identified and followed until medication stop date or the end of observation period. Patients were considered non-persistent whenever a permissible lag time of 6 months from the time of prescription issuance until medication filling date was exceeded. Treatment changes were based on physician decisions and patient preferences.

Demographic data including age, sex, body mass index (BMI), ethnicity, smoking history, and socioeconomic status as well as Charlson comorbidity index were retrieved. Data regarding use of steroids and conventional diseasemodifying anti-rheumatic drugs (CDMARDs) were also extracted. Descriptive statistics, including means (standard deviations) for continuous variables and frequencies (\%) for categorical variables, were used. Persistence estimates were derived using non-parametric survival analysis using Kaplan-Meier functions, with treatment discontinuations as failure events. Cox regression hazard ratio models were conducted to investigate factors associated with drug persistence.

Results: A total of 2301 PsA patients with 2958 treatment periods were identified and included in the analyses. Mean age of the study population was $50.9 \pm 14$ years, $54 \%$ were females, $70.4 \%$ were with $\mathrm{BMI}>25,40 \%$ were current smokers, and $76 \%$ were with a Charlson comorbidity index $>1$. The most commonly prescribed drug was etanercept (33\%), followed by adalimumab (29\%), golimumab (12\%), secukinumab (10\%), ustekinumab (8\%), and infliximab (8\%). While approximately $40 \%$ of patients persisted on therapy following 20 months of treatment, only about $20 \%$ of patients remained on any particular biologic agent after 5 years. Analyzing the data for all treatment periods while taking into account all lines of therapy revealed that secukinumab had a higher persistency than adalimumab, infliximab, and ustekinumab, with a log rank of $0.022,0.047$, and 0.001 , respectively. Female sex and smoking were associated with lower drug persistence ( $\mathrm{HR}=1.25,95 \% \mathrm{Cl}=1.13-1.38$ and $\mathrm{HR}=1.109,95 \% \mathrm{Cl}=1.01-$ 1.21 , respectively). On analyzing the data using only the first indicated biologic line, no superiority of any single anti-tumor necrosis factor-alpha (anti-TNFa) agent was observed, while secukinumab was found to be superior as (Continued on next page)

\footnotetext{
* Correspondence: Amirha3@clalit.org.il; devyzi@clalit.org.il

${ }^{1}$ Rheumatology Unit, Carmel Medical Center, 7 Michal Street, Haifa, Israel

Full list of author information is available at the end of the article
}

\section{BMC}

(c) The Author(s). 2021 Open Access This article is licensed under a Creative Commons Attribution 4.0 International License, which permits use, sharing, adaptation, distribution and reproduction in any medium or format, as long as you give appropriate credit to the original author(s) and the source, provide a link to the Creative Commons licence, and indicate if changes were made. The images or other third party material in this article are included in the article's Creative Commons licence, unless indicated otherwise in a credit line to the material. If material is not included in the article's Creative Commons licence and your intended use is not permitted by statutory regulation or exceeds the permitted use, you will need to obtain permission directly from the copyright holder. To view a copy of this licence, visit http://creativecommons.org/licenses/by/4.0/ The Creative Commons Public Domain Dedication waiver (http://creativecommons.org/publicdomain/zero/1.0/) applies to the data made available in this article, unless otherwise stated in a credit line to the data. 


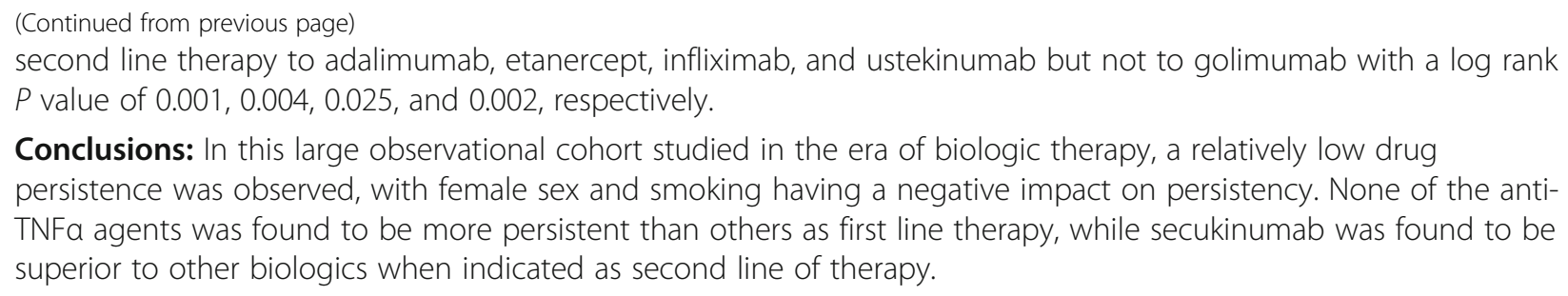

Keywords: Psoriatic arthritis, Drug persistence, Biologics

\section{Background}

Psoriatic arthritis is a seronegative spondyloarthropathy that greatly impacts patients' function and quality of life [1]. Beyond the musculoskeletal and skin features of the disease, patients with PsA experience fatigue, physical function limitations, sleep disturbance, and diminished work capacity and social participation [2].

In the past few decades, treatment of PsA has changed based on a better understanding of disease pathogenesis. Targeting TNF $\alpha$ is considered a milestone in the paradigm of PsA treatment as several randomized controlled trials and registries have demonstrated the efficacy of anti-TNF $\alpha$ agents in halting clinical and radiographic progression [3]. More recently, the interleukin-23 (IL23) $/ \mathrm{T}_{\mathrm{h}} 17$ axis has also been shown to play an important role in PsA disease pathogenesis with the IL-12/23 p40 inhibitor (ustekinumab) and IL-17A inhibitors (secukinumab and ixekizumab) also playing a pivotal role in disease control [3]. The most recent 2019 European League Against Rheumatism (EULAR) recommendations for PsA treatment advise on use of either IL-17 inhibitors or TNF $\alpha$ blockers as first line biologic therapy for PsA patients [4].

As more treatment options for PsA are currently available on the market than ever before, describing treatment persistence from real-life experience is gaining in importance as it contributes to better-informed clinical decisions regarding therapeutic choices for PsA patients with the goals of optimizing symptom remission, promoting functional recovery, and reducing healthcare costs.

The aim of this study was to estimate the treatment persistence in a real-world setting of biologic agents prescribed to PsA patients as well as factors associated with improved persistence of these drugs.

\section{Methods}

Clalit Health Services (CHS), Israel's largest healthcare provider serving some 4.4 million members constituting $52 \%$ of Israel's population, maintains a database that receives continuous real-time input from pharmaceutical, medical, and administrative digital systems [5]. Designed for purposes of administrative and clinical management, the database is available for clinical studies. The quality and accuracy of the register are high, and the reliability of the diagnoses of PsA was estimated in another study and found to be high [6] with a positive predictive value, sensitivity, and specificity of $90.5 \%, 88.7 \%$, and $88.1 \%$, respectively.

All patients with the diagnosis of PsA above the age of 18 with at least two consecutive dispensed prescriptions with any available biologic agent indicated for PsA under the Israeli National Healthcare Drug Plan from January 1, 2002, until December 31, 2018, were identified and followed until medication stop date or the end of the observation period. Under the Israeli National Healthcare Drug Plan, treatment with biologic therapy for PsA is indicated after the failure of two conventional diseasemodifying anti-rheumatic drugs (cDMARDs). At the time of the study, six biologic agents were approved and available on the Israeli market for PsA, including adalimumab, etanercept, infliximab, golimumab, ustekinumab, and secukinumab. Treatment was considered nonpersistent whenever a 6-month interval from time of prescription of any biologic agent until the time of medication filling was exceeded provided patients were not started on a different biologic agent during that period, in order to prevent the counting of any short absences of treatment for reasons such as infection or surgery to be classified as non-persistency. This 6-month time interval was decided after analyzing the data to allow for a reasonable grace period for medication filling as a shorter grace period of 3 or 4 months might have resulted in a higher misclassification rate of nonpersistence.

Demographic data including age, sex, ethnicity (Jewish or Arab), smoking history (current or past smoking), and socioeconomic status (SES) at inception (determined according to the CHS categories of low, medium, and high, a classification system which has been shown to highly correlate with SES assigned by the Israel Central Bureau of Statistics) [7] were collected. The BMI and patients' Charlson comorbidity index were calculated from the CHS database. Data regarding the concomitant use of glucocorticosteroids (GC) and cDMARDs such as methotrexate (MTX), leflunomide (LEF), sulfasalazine 
Table 1 Demographics and characteristics of the study group

\begin{tabular}{llll}
\hline Patients' characteristics & & \multicolumn{2}{l}{$\boldsymbol{N}=\mathbf{2 3 0 1}$} \\
\hline Age & Male & \multicolumn{2}{l}{$50.9 \pm 14$ years } \\
Sex & Female & 502 & $46.1 \%$ \\
& Unknown & 6 & $53.9 \%$ \\
SES & Low & 275 & $30.7 \%$ \\
& Medium & 365 & $40.8 \%$ \\
& High & 248 & $27.7 \%$ \\
Ethnicity & Arab & 120 & $13.4 \%$ \\
& Jewish & 774 & $86.6 \%$ \\
BMI & $0-18.5$ & 21 & $2.3 \%$ \\
& $18.5-25$ & 249 & $27.3 \%$ \\
& $25-30$ & 304 & $33.4 \%$ \\
& $30+$ & 337 & $37.0 \%$ \\
Obesity & No & 589 & $63.3 \%$ \\
& Yes & 342 & $36.7 \%$ \\
Smoking status & No & 557 & $59.9 \%$ \\
& Yes & 373 & $40.1 \%$ \\
& 0 & 227 & $24.4 \%$ \\
& 1 & 372 & $40.0 \%$ \\
& 2 & 148 & $15.9 \%$ \\
& 3 & 103 & $11.1 \%$ \\
& $4+$ & 80 & $8.6 \%$ \\
\hline
\end{tabular}

BMI body mass index, SES socioeconomic status

(SSZ), and hydroxychloroquine (HCQ) were also extracted from the database.

Descriptive statistics, including means (standard deviations) for continuous variables and frequencies (\%) for categorical variables, were used. Persistence estimates were derived using non-parametric survival analysis using Kaplan-Meier functions, with treatment discontinuations as failure events. Patients were right-censored for death and end of data availability. Cox regression hazard ratio models were conducted to investigate factors associated with drug persistence including age, sex, smoking status, the Charlson comorbidity index, the use of cDMARDs, and the year of initial availability of each biologic agent on the Israeli market.

The study was approved by the Institutional Review Board of Carmel Medical Center, Haifa (CMC0014-14).

\section{Results}

A total of 2301 PsA patients were identified and included in the analyses after meeting the inclusion criteria of the study. Patients' characteristics are described in Table 1 . The mean age of the study population was $50.9 \pm 14$ years, $54 \%$ were females, $70.4 \%$ were with BMI $>25 \mathrm{~kg} / \mathrm{m}^{2}, 36 \%$ were considered obese $(\mathrm{BMI}>30$ $\left.\mathrm{kg} / \mathrm{m}^{2}\right), 40 \%$ were current smokers, and only $24 \%$ had a Charlson comorbidity index of 0 .

From 2002 to 2018, a total of 2958 treatment periods were identified. The disposition of the patients and of the treatment periods is described in Table 2. The most commonly prescribed drug was etanercept (33\%), followed by adalimumab (29\%) and infliximab in only $8 \%$ of the cases. Fewer prescriptions of other biologic agents partially due to later entry of these therapeutic agents into the Israeli drug market or due to physician/ patient preferences were noted.

The concomitant use of cDMARDs with bDMARDs is listed in Table 3. A notable decrease in the use of MTX is seen with the introduction of all biologics. However, patients on infliximab were more likely to stay on concomitant treatment with MTX. Oral/parenteral GC were prescribed at least once during $33-43 \%$ of the treatment periods.

A Kaplan-Mayer survival analysis with pairwise comparisons of all treatment choices with respect to lines of therapy was conducted. When analyzing the data for all treatment periods while taking into account all lines of therapy, secukinumab had a higher persistency than adalimumab, infliximab, and ustekinumab, with a log rank of $0.022,0.047$, and 0.001 , respectively, as is shown in Fig. 1. Comparisons of other treatment modalities were not significantly different in this model.

Table 2 Treatment periods and frequency in the study group

\begin{tabular}{|c|c|c|c|c|c|}
\hline \multicolumn{3}{|c|}{ Treatment periods } & \multicolumn{3}{|c|}{ Patients on biologics } \\
\hline & Treatment periods & Percentage of patients & & Number of patients & Percentage of patients \\
\hline Adalimumab & 848 & 28.7 & Adalimumab & 660 & 28.7 \\
\hline Etanercept & 989 & 33.4 & Etanercept & 643 & 27.9 \\
\hline Infliximab & 233 & 7.9 & Infliximab & 179 & 7.8 \\
\hline Ustekinumab & 240 & 8.1 & Ustekinumab & 199 & 8.6 \\
\hline Golimumab & 356 & 12.0 & Golimumab & 331 & 14.4 \\
\hline Secukinumab & 292 & 9.9 & Secukinumab & 289 & 12.6 \\
\hline Total & 2958 & 100.0 & Total & 2301 & 100.0 \\
\hline
\end{tabular}


Table 3 The concomitant use of cDMARD/steroid medications with biologics

\begin{tabular}{|c|c|c|c|c|c|c|c|c|c|c|c|c|c|c|}
\hline \multirow[b]{3}{*}{ Concomitant MTX } & \multirow[b]{3}{*}{ No } & \multicolumn{12}{|c|}{ Treatment periods } & \multirow{3}{*}{$\begin{array}{l}\boldsymbol{P} \\
\text { value }\end{array}$} \\
\hline & & \multicolumn{2}{|c|}{ Adalimumab } & \multicolumn{2}{|c|}{ Etanercept } & \multicolumn{2}{|c|}{ Infliximab } & \multicolumn{2}{|c|}{ Ustekinumab } & \multicolumn{2}{|c|}{ Golimumab } & \multicolumn{2}{|c|}{ Secukinumab } & \\
\hline & & 642 & $75.7 \%$ & 807 & $81.6 \%$ & 159 & $68.2 \%$ & 210 & $87.5 \%$ & 267 & $75.0 \%$ & 244 & $83.6 \%$ & \\
\hline & Yes & 206 & $24.3 \%$ & 182 & $18.4 \%$ & 74 & $31.8 \%$ & 30 & $12.5 \%$ & 89 & $25.0 \%$ & 48 & $16.4 \%$ & \\
\hline \multirow[t]{2}{*}{ Concomitant other cDMARDs } & No & 783 & $92.3 \%$ & 919 & $92.9 \%$ & 210 & $90.1 \%$ & 221 & $92.1 \%$ & 311 & $87.4 \%$ & 264 & $90.4 \%$ & 0.03 \\
\hline & Yes & 65 & $7.7 \%$ & 70 & $7.1 \%$ & 23 & $9.9 \%$ & 19 & $7.9 \%$ & 45 & $12.6 \%$ & 28 & $9.6 \%$ & \\
\hline \multirow[t]{2}{*}{ MTX before } & No & 163 & $19.2 \%$ & 177 & $17.9 \%$ & 47 & $20.2 \%$ & 43 & $17.9 \%$ & 44 & $12.4 \%$ & 40 & $13.7 \%$ & 0.03 \\
\hline & Yes & 685 & $80.8 \%$ & 812 & $82.1 \%$ & 186 & $79.8 \%$ & 197 & $82.1 \%$ & 312 & $87.6 \%$ & 252 & $86.3 \%$ & \\
\hline \multirow[t]{2}{*}{ cDMARDs before } & No & 322 & $38.0 \%$ & 319 & $32.3 \%$ & 106 & $45.5 \%$ & 103 & $42.9 \%$ & 101 & $28.4 \%$ & 102 & $34.9 \%$ & 0.001 \\
\hline & Yes & 526 & $62.0 \%$ & 670 & $67.7 \%$ & 127 & $54.5 \%$ & 137 & $57.1 \%$ & 255 & $71.6 \%$ & 190 & $65.1 \%$ & \\
\hline \multirow[t]{2}{*}{ GC } & No & 510 & $60.1 \%$ & 633 & $64.0 \%$ & 132 & $56.7 \%$ & 141 & $58.8 \%$ & 195 & $54.8 \%$ & 196 & $67.1 \%$ & 0.03 \\
\hline & Yes & 338 & $39.9 \%$ & 356 & $36.0 \%$ & 101 & $43.3 \%$ & 99 & $41.3 \%$ & 161 & $45.2 \%$ & 96 & $32.9 \%$ & \\
\hline
\end{tabular}

CDMARDs conventional disease-modifying anti-rheumatic drugs, GC glucocorticosteroids, MTX methotrexate

On analyzing the data using only the first indicated biologic line, no biologic agent was found to be superior to any other as is shown in Fig. 2. Of note, during the study period, the four TNF-alpha blockers etanercept, adalimumab, infliximab, and golimumab were the only medications of this class available to be used as first line agents on the Israeli market so the comparison did not include secukinumab or ustekinumab.
As all biologic agents were equally available for use as second line agents, a survival analysis was conducted censoring for medications used as second line agents, with results shown in Fig. 3. The analysis revealed superiority of secukinumab compared to adalimumab, etanercept, infliximab, and ustekinumab but not to golimumab with a $\log \operatorname{rank} P$ value of $0.001,0.004,0.025$, and 0.002 , respectively. Golimumab had a higher persistency than

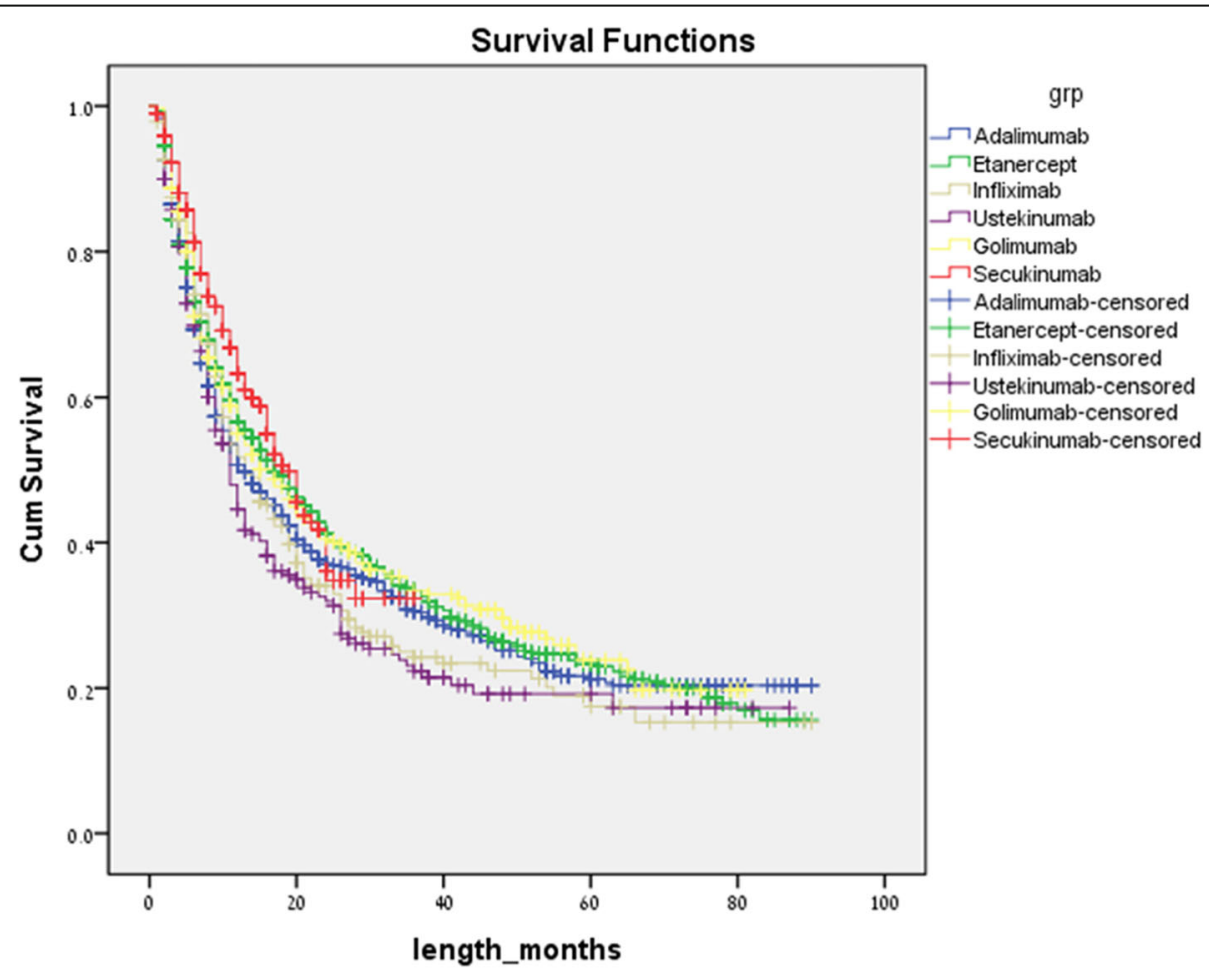

Fig. 1 Unadjusted time to non-persistence for all biologics used as all lines of therapy 


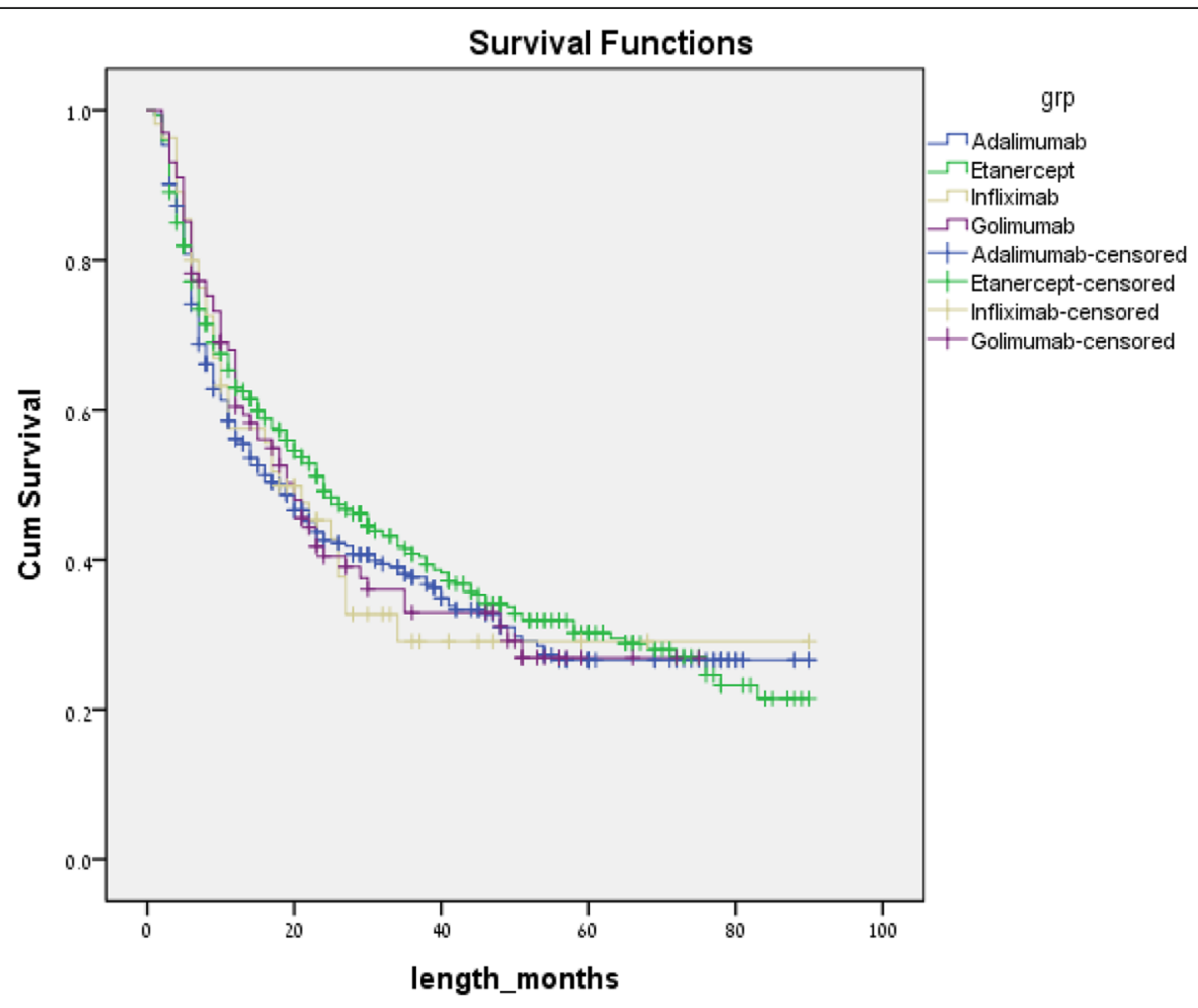

Fig. 2 Unadjusted time to non-persistence for biologics used as first lines of therapy

adalimumab with a $\log$ rank $P$ value of 0.027 . Other pairwise comparisons did not reach statistical difference.

Cox regression hazard ratio models were conducted to investigate factors associated with drug persistence as shown in Table 4. Female sex and smoking were associated with lower drug persistence. Patients who were obese also had lower estimates for drug persistency although not reaching statistical significance. Patients who were on concomitant treatment with MTX or GC were more likely to stay persistent on their biologic therapy. Moreover, a negative correlation was noted between drug persistency and the advancement of the various treatment lines, with a gradient decrease in drug persistency as patients advanced through alternative lines of biologic therapy, so that patients failing prior lines of treatment were less likely to stay persistent on newer lines of therapy.

Because time of introduction of any particular biologic agent into the Israeli drug market could potentially affect treatment choices and decisions, we incorporated the year of initial medication arrival onto the Israeli market into the statistical model, and compared it to 2018. In this analysis, we did not find any preference for any one medication over other medications for any given year. The 1-year, 2-year, and 5-year survival rate for biologics used in all lines of therapy ranging between $44.6-63.2 \%, 31.9-41.7 \%$, and $17.3-23.5 \%$ as reported in Table 5 .

\section{Discussion}

Real-world data on drug persistency in PsA patients is scarce. This study was conducted from a large, comprehensive, national dataset, composed of a diverse general population of 2301 PsA patients using all available bDMARDs indicated for PsA in Israel from 2002 until 2018.

We report higher drug persistency rates in PsA patients on secukinumab when indicated as second line therapy compared to adalimumab, infliximab, and ustekinumab. Our study also demonstrates improved biologic drug persistency in male PsA patients over female patients, with a reduction in drug persistency associated with smoking as well as biologic monotherapy.

Our real-world results on biologic persistence are in keeping with a study by Stober et al. [8] which evaluated the persistency of TNF $\alpha$ blockers limited to etanercept and adalimumab in 188 PsA patients, showing a lower persistency in female patients. As in that study, we did not find improved drug survival when analyzing the data for the first biologic agent used, which included the four 


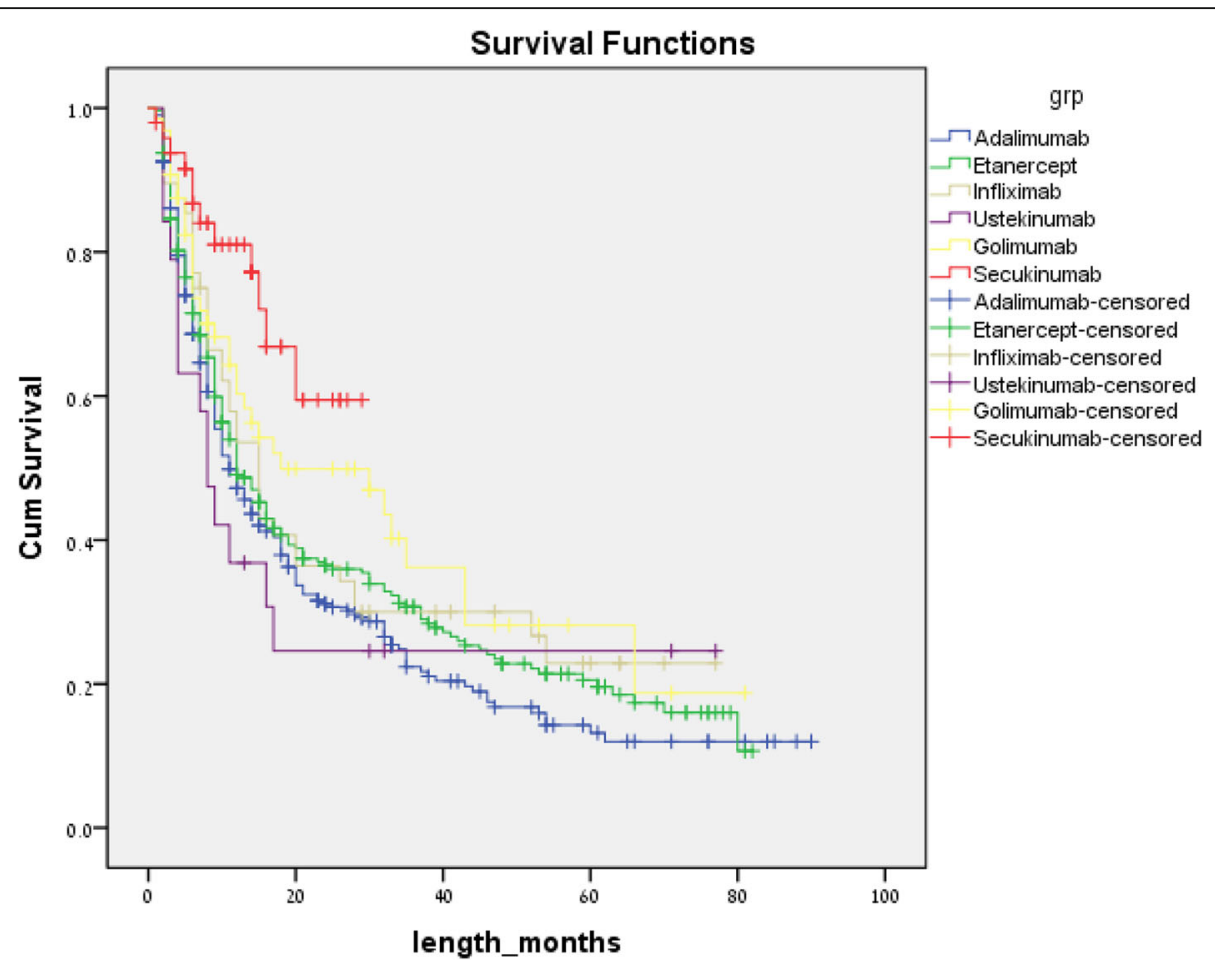

Fig. 3 Unadjusted time to non-persistence for biologics used as second line of therapy

TNF $\alpha$ blockers available on the market at the time of the study. As for the effect of MTX in combination therapy, we report a better drug persistency in patients on concurrent MTX. The role of MTX has been controversial, with a systemic review on all TNF $\alpha$ blockers failing to show any effect of combination therapy on drug survival except for infliximab [9], and with the SEAM study reporting no advantage for combination therapy of MTX with etanercept over monotherapy with etanercept alone [10]. However, drugs targeting the IL-17 pathway had similar efficacy in monotherapy or in combination with MTX as was shown in the FUTURE2, SPIRIT H2H, and EXCEED clinical trials data [11-13].

Our study did not find any association between patient age and SES on drug persistency. Our finding that smoking is a predictor for non-persistency is supported by other observational studies in the literature [14-16], as tobacco use is considered a significant environmental risk factor for developing inflammatory arthritis and there are indications that smoking exacerbates the symptoms and worsens disease outcomes. As for obesity, evidence from the literature points to its being a major culprit for disease non-response [17]. Findings from our study also support this notion, as there was a trend for lower drug persistency in obese patients, but this finding did not reach statistical significance.
As expected, patients who failed prior biologic therapy were less likely to remain persistent on newer biologic agents, in keeping with the report of Harrold et al. on data from the Corrona Registry on 549 biologic-naïve and 692 biologic-experienced PsA patients showing a greater mean time to nonpersistence in the former group [18]. In addition, numerous studies have demonstrated that switching between TNFa blockers results in lower response rates than when used as first line therapy $[19,20]$.

Our study showed that the persistence of golimumab was better than adalimumab when censoring the data for the second biologic line used. This is in keeping with the results of a study by Rotar et al. [21] on 2022 patients with either rheumatoid arthritis (RA), ankylosing spondylitis (AS), or PsA, which showed better persistence of golimumab compared to other TNF $\alpha$ blockers in biologic-experienced AS and PsA patients but not overall.

Our results report a 1-year, 2-year, and 5-year survival rate for biologics used in all lines of therapy ranging between $44.6-63.2 \%, 31.9-41.7 \%$, and $17.3-23.5 \%$, respectively, marginally lower compared to previous reports. In our review of the literature, the definitions used for drug persistency vary considerably in different studies. Saad et al. [22] showed that approximately $82 \%, 70 \%$, and $59 \%$ 
Table 4 Adjusted Cox proportional hazard model for treatment discontinuation

\begin{tabular}{|c|c|c|c|}
\hline & HR & $95.0 \%$ & \\
\hline & & Lower & Upper \\
\hline Female vs. male & 1.249 & 1.134 & 1.376 \\
\hline Age 30-39 (compared to 18-29) & 0.972 & 0.785 & 1.204 \\
\hline Age 40-49 & 1.000 & 0.810 & 1.234 \\
\hline Age 50-59 & 1.032 & 0.836 & 1.273 \\
\hline Age 60-69 & 0.945 & 0.758 & 1.178 \\
\hline Age $>70$ & 1.061 & 0.812 & 1.387 \\
\hline SES—medium & 0.898 & 0.798 & 1.011 \\
\hline SES—high & 0.918 & 0.805 & 1.048 \\
\hline Ethnicity—Arab & 1.136 & 0.972 & 1.328 \\
\hline Ethnicity—Jewish & 0.913 & 0.693 & 1.203 \\
\hline Charlson comorbidity index & 1.004 & 0.973 & 1.035 \\
\hline BMI_thin & 1.146 & 0.833 & 1.577 \\
\hline BMI_overweight & 1.082 & 0.952 & 1.228 \\
\hline BMI_obese & 1.126 & 0.993 & 1.278 \\
\hline Smokers & 1.109 & 1.008 & 1.219 \\
\hline Concomitant MTX & 0.830 & 0.737 & 0.933 \\
\hline Concomitant cDMARDs & 0.958 & 0.811 & 1.131 \\
\hline MTX use before & 0.891 & 0.788 & 1.008 \\
\hline cDMARDs use before & 1.027 & 0.931 & 1.134 \\
\hline Concurrent GC & 0.569 & 0.515 & 0.629 \\
\hline Rx line2 vs. line1 & 1.263 & 1.117 & 1.427 \\
\hline Rx line3 vs. line 1 & 1.296 & 1.119 & 1.500 \\
\hline Rx line4 vs. line 1 & 1.590 & 1.382 & 1.829 \\
\hline Start of biologic 2012 vs. 2018 & 1.657 & 1.339 & 2.051 \\
\hline Start of biologic 2013 vs. 2018 & 1.526 & 1.234 & 1.887 \\
\hline Start of biologic 2014 vs. 2018 & 1.484 & 1.204 & 1.829 \\
\hline Start of biologic 2015 vs. 2018 & 1.521 & 1.233 & 1.878 \\
\hline Start of biologic 2016 vs. 2018 & 1.697 & 1.393 & 2.066 \\
\hline Start of biologic 2017 vs.2018 & 1.410 & 1.157 & 1.719 \\
\hline
\end{tabular}

$B M I$ body mass index, $C D M A R D s$ conventional disease-modifying antirheumatic drugs, GC glucocorticosteroids, MTX methotrexate, Rx treatment, SES socioeconomic status of the population from the British Society of Rheumatology Biologics Register involving 566 participants with PsA remained on the first anti-TNF $\alpha$ agent after 1, 2, and 3 years of treatment, respectively. In 2016, Palmer and colleagues [23] observed that the mean biologic drug persistence was approximately 17 months among 990 PsA patients receiving TNF $\alpha$ blockers as first line therapy. In a different study, Fagerli et al. [24] estimated that approximately $47 \%$ of 625 participants diagnosed with PsA from the UK remained on their initial antiTNF $\alpha$ therapy after 5 years. Another study from an administrative claims database in the USA [25] on 1235 PsA patients reported $56 \%$ of patients discontinuing their index biologic agent within a year, with a mean duration of persistence of 8 months. More recently, Jacob and colleagues [26] reported a low persistence of biologics of $32 \%$ after 5 years of follow-up on PsA patients in rheumatology practices in Germany. Yet another recent study by the EuroSpa collaboration registries from Europe reported a 1-year retention rate of $77 \%$ for TNF $\alpha$ inhibitors in over 14,000 biologic-naïve PsA patients compared to a 2-year drug survival of 56\% and $50 \%$ for PsA patients on second line biologic therapy in the NOR-DMARD study [27] and in the Portuguese registry [28], respectively. Of note, it is important to emphasize that the definition of persistency in these studies was not similar, and the study populations, insurance coverages, and line of treatment reported differ as well precluding a direct comparison among the studies.

In our study, secukinumab had a better persistency when indicated as second line therapy. A number of matching-adjusted indirect comparisons have been published demonstrating conflicting though higher responses for secukinumab over infliximab [29], adalimumab [30], and etanercept [31]. Notably, secukinumab only narrowly missed statistical significance for superiority over adalimumab in the primary endpoint of the head-to-head EXCEED trial, showing only numerically higher results versus adalimumab [13]. Thus, our data might suggest that switching to secukinumab would be a better medication choice after TNF $\alpha$ blocker failure. This is more in line with the 2015 Group for Research and Assessment of Psoriasis and Psoriatic Arthritis (GRAPPA) treatment recommendations [3] and the

Table 5 The overall survival of biologic DMARDs

\begin{tabular}{llll}
\hline & 1-year survival (\%) & 2-year survival (\%) & 5-year survival (\%) \\
\hline Adalimumab & 50.7 & 37 & 21.3 \\
Etanercept & 56.4 & 41.2 & 23.3 \\
Infliximab & 51.7 & 34 & 17.5 \\
Golimumab & 55 & 40.5 & 22.5 \\
Ustekinumab & 44.6 & 31.9 & 17.3 \\
Secukinumab & 63.2 & 41.7 & - \\
\hline
\end{tabular}


updated 2019 EULAR recommendations [4] which place secukinumab as a first line agent alongside other biologics, unlike the earlier 2015 EULAR [32] as well as the 2018 American College of Rheumatology (ACR)/National Psoriasis Foundation (NPF) [33] guidelines, which specified TNF $\alpha$ blockers as first line biologic therapy and anti-IL-17 agents to be considered when TNF $\alpha$ blockers are "inappropriate."

There are some limitations in our study. Our results should be interpreted in caution as treatment indications and switching were not based on any protocol but rather on patient-physician choices and preferences. Moreover, we did not have any information on disease activity parameters involving joints and skin, nor any data on side effects that could have warranted medication switch and might have shed more light on the reason for treatment change and relatively low persistency rates.

On the other hand, the strengths of our study lie in our large dataset on PsA patients, providing a real-life image of drug treatment patterns and persistency as well as predictors for drug persistency.

In summary, our study of a large observational PsA cohort found a relatively low persistence of biologic therapy in rheumatology practices, with female sex and tobacco use having a negative impact on drug persistency, and secukinumab being superior to other biologic agents when indicated at second line of therapy. These findings can improve treatment planning and provide a more efficient allocation of societal economic resources. However, further studies are needed to establish the role and level of anti-IL-17 agents in the PsA treatment paradigm and to improve our understanding of the reasons for non-persistence.

\section{Abbreviations}

CASPAR: Classification Criteria for Psoriatic Arthritis; CDMARDs/ bDMARDs: Conventional/biologic disease-modifying anti-rheumatic drugs; IL: Interleukin; PsA: Psoriatic arthritis; TNFa: Tumor necrosis factor-al pha

\section{Acknowledgements}

Not applicable.

\section{Authors' contributions}

IF performed the statistical analysis; $T G, J F$, and IB reviewed and edited the paper; $\mathrm{AH}, \mathrm{DZ}$, and $\mathrm{AC}$ designed the work; $\mathrm{AH}, \mathrm{DZ}$, and FT analyzed and interpreted the data; $\mathrm{AH}$ and $\mathrm{DZ}$ drafted the paper. The authors read and approved the final manuscript.

\section{Funding}

This research was funded by a grant provided by the Israeli Rheumatology Association.

\section{Availability of data and materials}

All data generated or analyzed during this study are included in this published article.

\section{Ethics approval and consent to participate}

The research was reviewed and approved by the local Institutional Review Board at Carmel Medical Center (CMC-0014-14).
Consent for publication

Not applicable.

\section{Competing interests}

The authors declare that they have no competing interests.

\section{Author details}

${ }^{1}$ Rheumatology Unit, Carmel Medical Center, 7 Michal Street, Haifa, Israel. ${ }^{2}$ Chief Physician's Office, Central Headquarters, Clalit Health Services, Tel Aviv, Israel. ${ }^{3}$ Siaal Research Center for Family Medicine and Primary Care, Faculty of Health Sciences, Ben-Gurion University of the Negev, Beer-Sheba, Israel. ${ }^{4}$ Biostatistics unit, Carmel Medical Center, Haifa, Israel. ${ }^{5}$ Internal Medicine Department, Carmel Medical Center, Haifa, Israel. ${ }^{6}$ Ruth and Bruce Rappaport Faculty of Medicine, Technion-Israel Institute of Technology, Haifa, Israel.

Received: 14 September 2020 Accepted: 4 January 2021

Published online: 29 January 2021

\section{References}

1. Walsh JA, McFadden ML, Morgan MD, Sawitzke AD, Duffin KC, Krueger GG, et al. Work productivity loss and fatigue in psoriatic arthritis. J Rheumatol. 2014:41(8):1670-4.

2. Orbai A-M, de Wit M, Mease P, Shea JA, Gossec L, Leung YY, et al. International patient and physician consensus on a psoriatic arthritis core outcome set for clinical trials. Ann Rheum Dis. 2017;76(4):673-80.

3. Ogdie A, Coates LC, Gladman DD. Treatment guidelines in psoriatic arthritis. Rheumatology. 2020;59(Supplement_1):i37-46.

4. Gossec L, Baraliakos X, Kerschbaumer A, de Wit M, Mclnnes I, Dougados M, et al. EULAR recommendations for the management of psoriatic arthritis with pharmacological therapies: 2019 update. Ann Rheum Dis. 2020;79(6): $700-12$.

5. Vinker S, Fogelman Y, Elhayany A, Nakar S, Kahan E. Usefulness of electronic databases for the detection of unrecognized diabetic patients. Cardiovasc Diabetol. 2003;2:13.

6. Eder L, Cohen AD, Feldhamer I, Greenberg-Dotan S, Batat E, Zisman D. The epidemiology of psoriatic arthritis in Israel - a population-based study. Arthritis Res Ther. 2018:20(1):3.

7. Filc D, Davidovich N, Novack L, Balicer RD. Is socioeconomic status associated with utilization of health care services in a single-payer universal health care system? Int J Equity Health. 2014;13:115.

8. Stober C, Ye W, Guruparan T, Htut E, Clunie G, Jadon D. Prevalence and predictors of tumour necrosis factor inhibitor persistence in psoriatic arthritis. Rheumatology. 2018;57(1):158-63.

9. Behrens F, Cañete JD, Olivieri I, van Kuijk AW, McHugh N, Combe B. Tumour necrosis factor inhibitor monotherapy vs combination with MTX in the treatment of PsA: a systematic review of the literature. Rheumatology. 2015; 54(5):915-26.

10. Mease PJ, Gladman DD, Collier DH, Ritchlin CT. Helliwell PS, Liu L, et al. Etanercept and methotrexate as monotherapy or in combination for psoriatic arthritis: primary results from a randomized, controlled phase III trial. Arthritis Rheumatol. 2019;71(7):1112-24.

11. McInnes IB, Mease PJ, Ritchlin CT, Rahman P, Gottlieb AB, Kirkham B, et al. Secukinumab sustains improvement in signs and symptoms of psoriatic arthritis: 2 year results from the phase 3 FUTURE 2 study. Rheumatology. 2017:56(11):1993-2003.

12. Mease PJ, Smolen JS, Behrens F, Nash P, Liu Leage S, Li L, et al. A head-tohead comparison of the efficacy and safety of ixekizumab and adalimumab in biological-naïve patients with active psoriatic arthritis: 24-week results of a randomised, open-label, blinded-assessor trial. Ann Rheum Dis. 2020;79(1): 123-31.

13. Mclnnes IB, Behrens F, Mease PJ, Kavanaugh A, Ritchlin C, Nash P, et al. Secukinumab versus adalimumab for treatment of active psoriatic arthritis (EXCEED): a double-blind, parallel-group, randomised, active-controlled, phase 3b trial. Lancet. 2020;395(10235):1496-505.

14. Højgaard P, Glintborg B, Hetland ML, Hansen TH, Lage-Hansen PR, Petersen $\mathrm{MH}$, et al. Association between tobacco smoking and response to tumour necrosis factor a inhibitor treatment in psoriatic arthritis: results from the DANBIO registry. Ann Rheum Dis. 2015;74(12):2130-6.

15. Viguier M, Livideanu C, Beylot-Barry M, Richard M-A, Paul C, Bachelez H, et al. Observational case series on a group of psoriasis patients who failed to respond to any TNF blockers. J Dermatolog Treat. 2014;25(1):75-7. 
16. Di Lernia V, Ricci C, Lallas A, Ficarelli E. Clinical predictors of non-response to any tumor necrosis factor (TNF) blockers: a retrospective study. J Dermatolog Treat. 2014;25(1):73-4.

17. Ogdie A, Palmer JL, Greenberg J, Curtis JR, Harrold LR, Solomon DH, et al. Predictors of achieving remission among patients with psoriatic arthritis initiating a tumor necrosis factor inhibitor. J Rheumatol. 2019:46(5):475-82.

18. Harrold LR, Stolshek BS, Rebello S, Collier DH, Mutebi A, Wade SW, et al. Impact of prior biologic use on persistence of treatment in patients with psoriatic arthritis enrolled in the US Corrona registry. Clin Rheumatol. 2017; 36(4):895-901.

19. Costa L, Perricone C, Chimenti MS, Del Puente A, Caso P, Peluso R, et al. Switching between biological treatments in psoriatic arthritis: a review of the evidence. Drugs R D. 2017;17(4):509-22.

20. Reddy SM, Crean S, Martin AL, Burns MD, Palmer JB. Real-world effectiveness of anti-TNF switching in psoriatic arthritis: a systematic review of the literature. Clin Rheumatol. 2016;35(12):2955-66.

21. Rotar Ž, Tomšič M, Praprotnik S, Slovenian Rheumatologists. The persistence of golimumab compared to other tumour necrosis factor-a inhibitors in daily clinical practice for the treatment of rheumatoid arthritis, ankylosing spondylitis and psoriatic arthritis: observations from the Slovenian nationwide longitudinal registry of patients treated with biologic diseasemodifying antirheumatic drugs-BioRx.si. Clin Rheumatol. 2019;38(2):297-305.

22. Saad AA, Ashcroft DM, Watson KD, Hyrich KL, Noyce PR, Symmons DPM, et al. Persistence with anti-tumour necrosis factor therapies in patients with psoriatic arthritis: observational study from the British Society of Rheumatology Biologics Register. Arthritis Res Ther. 2009;11(2):R52.

23. Palmer JB, Li Y, Herrera V, Liao M, Tran M, Ozturk ZE. Treatment patterns and costs for anti-TNFa biologic therapy in patients with psoriatic arthritis. BMC Musculoskelet Disord. 2016;17:261.

24. Fagerli KM, Kearsley-Fleet L, Watson KD, Packham J, Contributors Group B-R, Symmons DPM, et al. Long-term persistence of TNF-inhibitor treatment in patients with psoriatic arthritis. Data from the British Society for Rheumatology Biologics Register. RMD Open. 2018;4(1):e000596.

25. Walsh JA, Adejoro O, Chastek B, Palmer JB, Hur P. Treatment patterns among patients with psoriatic arthritis treated with a biologic in the United States: descriptive analyses from an administrative claims database. J Manag Care Spec Pharm. 2018;24(7):623-31.

26. Jacob L, Chevalier T, Kostev K. Persistence with biological drugs in patients treated in rheumatology practices in Germany. Rheumatol Int. 2019;39(3): 525-31.

27. Fagerli KM, Lie E, van der Heijde D, Heiberg MS, Kalstad S, Rødevand E, et al. Switching between TNF inhibitors in psoriatic arthritis: data from the NORDMARD study. Ann Rheum Dis. 2013;72(11):1840-4.

28. Vieira-Sousa E, Eusébio M, Ávila-Ribeiro P, Khmelinskii N, Cruz-Machado R, Rocha TM, Bernardes M, Santos-Faria D, Silva JL, Santos H, Miguel C, Carvalho P, Costa T, Duarte AC, Meirinhos T, Nero P, Fonseca JE, Santos MJ. Real-world longterm effectiveness of tumor necrosis factor inhibitors in psoriatic arthritis patients from the Rheumatic Diseases Portuguese Register. J Rheumatol. 2020;47(5):690-700. https://doi.org/10.3899/jrheum.181272.

29. Strand V, Mclnnes I, Mease P, Nash P, Thom H, Kalyvas C, et al. Matchingadjusted indirect comparison: secukinumab versus infliximab in biologicnaive patients with psoriatic arthritis. J Comp Eff Res. 2019;8(7):497-510.

30. Nash P, Mclnnes IB, Mease PJ, Thom H, Hunger M, Karabis A, et al. Secukinumab versus adalimumab for psoriatic arthritis: comparative effectiveness up to 48 weeks using a matching-adjusted indirect comparison. Rheumatol Ther. 2018;5(1):99-122.

31. Mease P, Choy E, Nash P, Kalyvas C, Hunger M, Pricop L, et al. Comparative effectiveness of secukinumab and etanercept in biologic-naïve patients with psoriatic arthritis assessed by matching-adjusted indirect comparison. Eur J Rheumatol. 2018;6(3):113-21.

32. Gossec L, Smolen JS, Ramiro S, de Wit M, Cutolo M, Dougados M, et al. European League Against Rheumatism (EULAR) recommendations for the management of psoriatic arthritis with pharmacological therapies: 2015 update. Ann Rheum Dis. 2016;75(3):499-510.

33. Singh JA, Guyatt G, Ogdie A, Gladman DD, Deal C, Deodhar A, et al. Special article: 2018 American College of Rheumatology/National Psoriasis Foundation guideline for the treatment of psoriatic arthritis. Arthritis Rheumatol. 2019;71(1):5-32.

\section{Publisher's Note}

Springer Nature remains neutral with regard to jurisdictional claims in published maps and institutional affiliations.
Ready to submit your research? Choose BMC and benefit from:

- fast, convenient online submission

- thorough peer review by experienced researchers in your field

- rapid publication on acceptance

- support for research data, including large and complex data types

- gold Open Access which fosters wider collaboration and increased citations

- maximum visibility for your research: over $100 \mathrm{M}$ website views per year

At $\mathrm{BMC}$, research is always in progress.

Learn more biomedcentral.com/submissions 